\title{
SUSCEPTIBILITY TESTS OF OROPHARYNGEAL CANDIDA ALBICANS FROM EGYPTIAN PATIENTS TO FLUCONAZOLE DETERMINED BY THREE METHODS
}

\author{
Noha El-Mashad, Mona F. Foad, Niveen Saudy, Dalia A. Salem \\ Department of Clinical Pathology, Faculty of Medicine, Mansoura University, Egypt.
}

Submitted: December 07, 2010; Returned to authors for corrections: May 05, 2011; Approved: August 15, 2011.

\begin{abstract}
Candida albicans frequently cause oropharyngeal candidiasis in immunocompromised patients. As some of these isolates show resistance against azoles, the clinician is wary of initiating therapy with fluconazole (FZ) until a final susceptibility report is generated. We aimed to evaluate the efficacy of rapid flow cytometry (FCM) and disc diffusion (DD) methods in comparison to reference microdilution (MD) of Clinical and Laboratory Standards Institute (CLSI) method for FZ. Thirty seven Candida albicans isolates were tested by the three methods. By both MD and FCM, 26/37 (70.3\%) were sensitive with minimal inhibitory concentration (MIC) $\leq 8 \mu \mathrm{g} / \mathrm{ml}, 5 / 37$ (13.5\%) were susceptible dose dependant (S-DD) with MIC 16-32 $\mu \mathrm{g} / \mathrm{ml}$ and $6 / 37(16.2 \%)$ were resistant with MIC $\geq 64 \mu \mathrm{g} / \mathrm{ml}$. More than $92 \%$ of isolates susceptible to FZ by the MD were susceptible by the DD methods with good agreement $(81.08 \%, P=0.000)$. However, $4 / 5$ isolates diagnosed as S-DD by MD were resistant by DD. Interestingly, the MIC by FCM at $4 \mathrm{~h}$ showed excellent agreement $(95.59 \%, P=0.000)$ to that obtained by MD method at $24 \mathrm{~h}$. Overall, FCM antifungal susceptibility testing provided rapid, reproducible results that are valuable alternative to MD. The DD test is recommended as a simple and reliable screening test for the detection of susceptible Candida albicans isolates to FZ.
\end{abstract}

Key word: CLSI, Disk diffusion, flowcytometry, fluconazole, malignant patients, NCCLS

\section{INTRODUCTION}

Oropharyngeal candidiasis is a common infection in cancer patients receiving therapies. The oral mucosal infection (ranging from 17 to 29\%) with Candida albicans are particularly common in onco-hematological patients resulted in a higher risk for developing invasive candidiasis with an attributable mortality around $40 \%$ (23).
Fluconazole (FZ), a well-tolerated and safe triazole antifungal agent that distributes widely in body tissues and is active against the major fungal pathogens in immunocompromized patients with few side effects. Its empiric use in onco-haematological patients may result in a higher colonization rate with FZ-resistant Candida species (28). Careful periodical surveillance is needed in order to identify any changes in the susceptibility patterns of FZ with

*Corresponding Author. Mailing address: Lecturer of Clinical Pathology, Clinical Pathology Department, Faculty of Medicine, Mansoura University, Egypt.; Tel.: +20-10-8368989 Fax:+20-50-2248203.; E-mail: niveen197@ mans.edu.eg 
the increased use of this antifungal agent $(8,15)$.

The "Clinical and Laboratory Standards Institute" (CLSI; formerly NCCLS) Subcommittee on Antifungal Susceptibility Tests has developed reproducible procedures for the antifungal susceptibility testing of yeasts for fluconazole by broth microdilution MD (documents M27-A2) method (3, 6). Because this method is time consuming, difficult to perform and labor intensive, alternative rapid and reliable methods are needed (19). Agar based method as disk diffusion (DD) is attractive because of its simplicity and low-cost $(1,10)$. CLSI had also established a DD procedure of yeasts for FZ (document M44-A) (7). Several years ago, it was suggested that flow cytometry (FCM) might be useful for susceptibility testing of micro-organisms, as drug-induced cell damage could be assessed by use of various fluorescent dyes on a cell-by-cell basis $(20,24)$

A rapid reliable susceptibility testing method would enable the clinician to prescribe the most suitable antifungal agent, thus avoiding more toxic or expensive therapy. In this aspect we aimed to evaluate the efficacy of rapid FCM and DD in comparison to reference MD method for FZ susceptibility testing of Candida albicans isolates.

\section{MATERIALS AND METHODS}

\section{Microorganisms}

They were 37 Candida albicans isolated from recurrent oral candidiasis of onco-hematological patients. The patients were randomly selected from Out Patient Clinic of Oncology Center, Mansoura University Hospital, Egypt. No patient was under treatment with antifungal drugs. Oral consents were obtained that were approved by ethical committee of Mansoura Faculty of Medicine, Egypt. The isolates were identified by morphology, microscopy, germ tube test and subjected to metabolic evaluation by use of API 20C system (Biomerieux, SA, Marcy-l'Etoile, France). Candida albicans isolates were stored in sterile distilled water in $50 \%$ glycerol at $-70^{\circ} \mathrm{C}$. Each isolate was subcultured twice on Sabouraud dextrose agar (Difco Laboratories, Detroit, USA) and incubated at $35^{\circ} \mathrm{C}$ to ensure purity and optimal growth. All isolates were subcultured again $24 \mathrm{~h}$ prior to testing (12).

\section{Antifungal Susceptibility testing}

Inoculum suspensions: Yeast inoculums suspensions were prepared as described in CLSI M27-A2. Briefly, for each isolate, 5 colonies greater than $1 \mathrm{~mm}$ in diameter were selected, suspended in $0.9 \%$ saline solution and adjusted to a final concentration of $0.5 \mathrm{Mc}$ Farland standard (Sigma, St. Louis, USA) with approximately $1-5 \times 10^{6} \mathrm{CFU} / \mathrm{ml}$. This suspension was used directly to inoculate agar plates for disk diffusion (DD) procedure and flow cytometry (FCM) procedure or was diluted for the microdilution (MD) procedure as following: the suspensions were further diluted to a ratio of 1:50 in $5 \mathrm{ml}$ sterile saline and then diluted to a ratio of 1:20 in RPMI 1640 to attain a final inoculum concentration in the titration wells of $1.5 \pm 1.0 \times 10^{3}$ cells $/ \mathrm{ml}$. Each dilution was vortexed for 30 seconds before proceeding $(6,16)$

Antifungal agents: The antifungal agent fluconazole (FZ) (SEDICO Pharmaceutical Co., 6 October City-Egypt) was used in the susceptibility tests. For MD and FCM procedures, FZ powder was dissolved in distilled water to yield stock solutions containing $6400 \mu \mathrm{g} / \mathrm{mL}$ and were stored at $-70^{\circ} \mathrm{C}$ in $400 \mu \mathrm{L}$ aliquots until used. Serial two fold dilutions of FZ were prepared with RPMI 1640 containing L-glutamine without bicarbonate, buffered to $\mathrm{pH} \quad 7.0$ with $0.165 \mathrm{M}$ morpholinepropane sulfonic acid (MOPS; Sigma). The final concentrations were 0.125 to $64 \mu \mathrm{g} / \mathrm{ml}$. For DD procedure, FZ

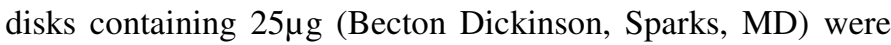
used.

\section{Procedures}

\section{1- Broth microdilution assay MD (document M27-A2):}

The susceptibility assays were performed in sterile 96-well round bottom microplates as described previously. For each 
drug concentration (from 0.125 to $64 \mu \mathrm{g} / \mathrm{m}$ ), $100 \mu \mathrm{l}$ were dispensed in microwells of titration plate. Then, $100 \mu \mathrm{l}$ of the prepared organism suspension was pipetted in each well reaching a final test volume of $200 \mu \mathrm{l}$. For each isolate, a growth control well contained yeast suspension and RPMI 1640 without FZ was prepared. After 24 hours of incubation at $35^{\circ} \mathrm{C}$, MIC (minimum inhibitory concentration) was determined visually by comparing its turbidity with the drugfree growth control well. The MIC was defined as the lowest drug concentration that cause $\geq 50 \%$ growth inhibition compared to drug-free growth control well. Final inoculum's size was confirmed by subculture and colony count. Isolates were classified as susceptible if the MIC for the isolate was $\leq 8$ $\mu \mathrm{g} / \mathrm{ml}$, susceptible-dose dependent (S-DD) if the MIC was 16 $32 \mu \mathrm{g} / \mathrm{ml}$, and resistant if the MIC was $\geq 64 \mu \mathrm{g} / \mathrm{ml}(4,6)$.

2- Flow Cytometry (FCM) method: Adjusted inocula of yeast cells killed by boiling at $100^{\circ} \mathrm{C}$ for $20 \mathrm{~min}$ were used as a staining control. Propidium iodide (PI) (Sigma, P4170-10MG, USA), a membrane-impermeable DNA-intercalating dye, was used to stain the yeast cells. Sodium deoxycholate (Sigma),a detergent, was used to facilitate diffusion of PI into the yeast cell membranes which were damaged by FZ. One-half milliliter of the yeast suspension (adjusted to 0.5 McFarland standard) was added to $0.5 \mathrm{ml}$ of serial two fold FZ dilutions and incubated at $35^{\circ} \mathrm{C}$ for $4 \mathrm{~h}$. The growth control tube received $0.5 \mathrm{ml}$ of drug diluting fluid without $\mathrm{FZ}$. At the end of incubation, $200 \mu \mathrm{l}$ of each mixture was placed in 12 - by 75 $\mathrm{mm}$ polystyrene tubes. Two hundred microliters of $25 \mathrm{mM}$ sodium deoxy-cholate and $10 \mu \mathrm{l}$ of $1 \%$ PI were added to each dilution. The tubes were mixed by flicking with fingers and incubated for $5 \mathrm{~min}$ at room temperature in the dark before FCM analysis. The samples were analyzed with a FAC Scan flow cytometry (Becton Dickinson, Lincoln Park, N.J, USA.) and Cell Quest software for data acquisition and analysis. Ten thousand yeast cells were analyzed for forward scatter (3.73 linear gain), side scatter $(270-\mathrm{V} \log ), \log$ of red fluorescence, FL2 (457-V log), threshold value 52, and mean channel fluorescence (MCF; intensity of fluorescence of yeasts labeled with PI). Controls included samples containing viable cells, heat-killed cells, cells with sodium deoxycholate, and cells with PI and sodium deoxycholate. The MIC was defined as the lowest concentration of drug that showed an increase of $50 \%$ in MCF compared to that of the growth control $(12,22)$.

\section{3- Disk diffusion procedure DD (document M44-A):}

The adjusted inocula to $0.5 \mathrm{McFarland}$ standard and test disks contained $25 \mu \mathrm{g}$. FZ were used. A sterile cotton swab was dipped in the suspension and streaked in many directions over the entire surface of the plate containing Mueller-Hinton agar supplemented with $2 \%$ glucose and $0.5 \mu \mathrm{g} / \mathrm{ml}$ methylene blue (due to the ability of that medium to produce enhanced definition of growth margins). The plates were allowed to dry for 5-15 minutes before the FZ discs were applied on the agar plates. They were then incubated at $35^{\circ} \mathrm{C}$ for $24 \mathrm{~h}$. The inhibition zone sizes were measured in millimeters. The isolates with the zone diameters of $\geq 19 \mathrm{~mm}$ were reported as sensitive to FZ, those of $15-18 \mathrm{~mm}$ as susceptible-dosedependant (S-DD) while the ones $\leq 14 \mathrm{~mm}$ were reported to be resistant $(5,10)$.

\section{Statistical analysis}

Reliability and reproducibility were assessed with the use of Kappa coefficient test. It represents the percentage of instances of agreement between MD method (standard) and either disc diffusion or flowcytometry. It was considered agreement when MIC results of test and MD method were in exact agreement. $P<0.05$ is significant and $P<0.001$ is highly significant.

\section{RESULTS}

MICs of fluconazole (FZ) by broth micro-dilution (MD) method were determined for 37 Candida albicans isolates after $24 \mathrm{~h}$ using the CLSI guidelines. Six of them (16\%) were found to be resistant and 5 (13.5\%) were S-DD (Table1). 
Table 2 shows the comparison of results for all isolates by within two drug dilutions of those of the reference method for the MD and FCM analysis for FZ. It revealed that MICs were most isolates.

Table 1. Susceptibility of C. albicans to fluconazole through broth microdilution, disc diffusion and flow cytometry".

\begin{tabular}{|c|c|c|c|}
\hline \multirow[b]{2}{*}{ Strain } & \multicolumn{3}{|c|}{ Susceptibility tests } \\
\hline & $\begin{array}{l}\text { broth microdilution } \\
\text { MIC }(24 \mathrm{~h})(\mu \mathrm{g} / \mathrm{ml})\end{array}$ & $\begin{array}{c}\text { Flow cytometry } \\
\text { MICs }(4 \mathrm{~h})(\mu \mathrm{g} / \mathrm{ml})\end{array}$ & $\begin{array}{c}\text { Disc Diffusion } \\
\text { inhibition halo }(\mathbf{m m})\end{array}$ \\
\hline 1 & 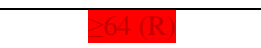 & $64(\mathrm{R})$ & $<14(\mathrm{R})$ \\
\hline 2 & $0.25(\mathrm{~S})$ & $0.5(\mathrm{~S})$ & $30(\mathrm{~S})$ \\
\hline 3 & $0.5(\mathrm{~S})$ & $0.5(\mathrm{~S})$ & $34(\mathrm{~S})$ \\
\hline 4 & 32 (SDD) & 16(SDD) & $<14(\mathrm{R})$ \\
\hline 5 & $4(\mathrm{~S})$ & $2(\mathrm{~S})$ & $26(S)$ \\
\hline 6 & $\geq 64(\mathrm{R})$ & $32(\mathrm{SDD})$ & $<14(\mathrm{R})$ \\
\hline 7 & $4(\mathrm{~S})$ & $2(\mathrm{~S})$ & $33(\mathrm{~S})$ \\
\hline 8 & $0.25(\mathrm{~S})$ & $0.5(\mathrm{~S})$ & $28(\mathrm{~S})$ \\
\hline 9 & $\geq 64(\mathrm{R})$ & 64(R) & $<14(\mathrm{R})$ \\
\hline 10 & $0.5(\mathrm{~S})$ & $1(\mathrm{~S})$ & $28(\mathrm{~S})$ \\
\hline 11 & $0.5(\mathrm{~S})$ & $0.25(\mathrm{~S})$ & $30(\mathrm{~S})$ \\
\hline 12 & $8(\mathrm{~S})$ & $8(\mathrm{~S})$ & $<14(\mathrm{R})$ \\
\hline 13 & $0.25(\mathrm{~S})$ & $0.25(\mathrm{~S})$ & $25(\mathrm{~S})$ \\
\hline 14 & $0.25(\mathrm{~S})$ & $0.125(\mathrm{~S})$ & $22(\mathrm{~S})$ \\
\hline 15 & $0.5(\mathrm{~S})$ & $0.25(\mathrm{~S})$ & $30(\mathrm{~S})$ \\
\hline 16 & 16(SDD) & 16(SDD) & 17(SDD) \\
\hline 17 & $0.125(\mathrm{~S})$ & $0.25(\mathrm{~S})$ & $24(\mathrm{~S})$ \\
\hline 18 & $\geq 64(\mathrm{R})$ & $64(\mathrm{R})$ & $<14(\mathrm{R})$ \\
\hline 19 & $0.5(\mathrm{~S})$ & $1(\mathrm{~S})$ & $32(\mathrm{~S})$ \\
\hline 20 & $0.5(\mathrm{~S})$ & $0.5(\mathrm{~S})$ & $26(\mathrm{~S})$ \\
\hline 21 & 16(SDD) & 32 (SDD) & $<14(\mathrm{R})$ \\
\hline 22 & $1(\mathrm{~S})$ & $4(\mathrm{~S})$ & $20(S)$ \\
\hline 23 & $0.5(\mathrm{~S})$ & $0.25(\mathrm{~S})$ & $26(S)$ \\
\hline 24 & $0.25(\mathrm{~S})$ & $0.5(\mathrm{~S})$ & $22(\mathrm{~S})$ \\
\hline 25 & $0.125(\mathrm{~S})$ & $0.25(\mathrm{~S})$ & $30(\mathrm{~S})$ \\
\hline 26 & $0.25(\mathrm{~S})$ & $0.25(\mathrm{~S})$ & $27(\mathrm{~S})$ \\
\hline 27 & $\geq 64(\mathrm{R})$ & 64(R) & $<14(\mathrm{R})$ \\
\hline 28 & $0.125(\mathrm{~S})$ & $0.125(\mathrm{~S})$ & $29(\mathrm{~S})$ \\
\hline 29 & $2(\mathrm{~S})$ & $2(\mathrm{~S})$ & $25(\mathrm{~S})$ \\
\hline 30 & 32 (SDD) & 32 (SDD) & $<14(\mathrm{R})$ \\
\hline 31 & $0.5(\mathrm{~S})$ & $1(\mathrm{~S})$ & $22(\mathrm{~S})$ \\
\hline 32 & $32(\mathrm{SDD})$ & $32(\mathrm{SDD})$ & $<14(\mathrm{R})$ \\
\hline 33 & $1(\mathrm{~S})$ & $1(\mathrm{~S})$ & $30(\mathrm{~S})$ \\
\hline 34 & E64(R) & 32 (SDD) & 15 (SDD) \\
\hline 35 & $0.125(\mathrm{~S})$ & $0.25(\mathrm{~S})$ & $27(\mathrm{~S})$ \\
\hline 36 & $8(\mathrm{~S})$ & $8(\mathrm{~S})$ & 15(SDD) \\
\hline 37 & $0.5(\mathrm{~S})$ & $2(\mathrm{~S})$ & $30(\mathrm{~S})$ \\
\hline
\end{tabular}


Table 2. Flowcytometry compared to the MD reference method for Fluconazole susceptibility

\begin{tabular}{|c|c|c|c|c|c|c|c|c|c|c|c|}
\hline & \multicolumn{11}{|c|}{$\begin{array}{l}\text { MIC distribution of Fluconazole by MD } \\
\qquad(\mu \mathrm{g} / \mathrm{ml})\end{array}$} \\
\hline & \multicolumn{7}{|c|}{ Sensitive } & \multicolumn{2}{|c|}{ Intermed } & \multicolumn{2}{|c|}{ Resist } \\
\hline & 0.125 & 0.25 & 0.5 & 1.0 & 2.0 & 4.0 & 8.0 & $\underline{16}$ & $\underline{32}$ & $\geq 64$ & Total \\
\hline & 4 & 6 & 9 & 2 & 1 & 2 & 2 & 2 & 3 & 6 & 37 \\
\hline $\begin{array}{l}\text { Flow } \\
\text { cytometry }\end{array}$ & 2 & 8 & 5 & 4 & 4 & 1 & 2 & 2 & 5 & 4 & 37 \\
\hline
\end{tabular}

Also, disc diffusion (DD) testing of all Candida isolates to FZ was performed. On measuring the inhibition zone diameters of $25 \mu \mathrm{g} \mathrm{FZ} \mathrm{disk} \mathrm{for} \mathrm{the} 6$ resistant strains by MD, 5 (83\%) had confirmed to be resistant by zone diameters of $<14 \mathrm{~mm}$ while the remaining strain was of S-DD with a zone diameter 15 $\mathrm{mm}$. Moreover, out of the 5 S-DD strains, only $1(20 \%)$ confirmed to be S-DD with zone diameter of $16 \mathrm{~mm}$ and.4 $(80 \%)$ were resistant with zone diameters of $<14 \mathrm{~mm}$.
Interestingly, among a total of 26 strains found to be susceptible by the MD method, 2 (7.7\%) yielded FZ zone sizes of $<19 \mathrm{~mm}$ and would have been misclassified as resistant or S-DD strains on the basis of the disc test (Table 3).

The levels of agreement between the MD and either DD or FCM (Table 4) showed that FCM was of excellent agreement while DD showed good agreement with a highly significant correlation $(P ; 0.000)$

Table 3. Disk Diffusion compared to the MD reference method for Fluconazole susceptibility

\begin{tabular}{|c|c|c|c|c|c|c|c|c|c|c|c|c|}
\hline & & \multicolumn{11}{|c|}{$\begin{array}{c}\text { MIC distribution of Fluconazole by MD } \\
(\mu \mathrm{g} / \mathrm{ml})\end{array}$} \\
\hline & & \multicolumn{7}{|c|}{ Sensitive } & \multicolumn{2}{|c|}{ Intermed } & \multicolumn{2}{|l|}{ Resist } \\
\hline & & 0.125 & 0.25 & 0.5 & 1.0 & 2.0 & 4.0 & 8.0 & 16 & 32 & $\geq 64$ & Total \\
\hline & & 4 & 6 & 9 & 2 & 1 & 2 & 2 & 2 & 3 & 6 & 37 \\
\hline Disc diffusion test & $R(0-14)$ & & & & & & & 1 & 1 & 3 & 5 & 10 \\
\hline Zone diamter & I $(15-18)$ & & & & & & & 1 & 1 & & 1 & 3 \\
\hline$(\mathbf{m m})$ & $S(\geq 19)$ & 4 & 6 & 9 & 2 & 1 & 2 & & & & & 24 \\
\hline
\end{tabular}

Table 4. Overall agreement between results of fluconazole susceptibility tests and of standard 24-h broth MD reference test

\begin{tabular}{|c|c|c|c|c|}
\hline Test & $\begin{array}{c}\text { Kappa } \\
\text { Coefficient }\end{array}$ & Level of agreement & $\begin{array}{c}\text { Percentage } \\
\text { Agreement }^{\text {b }}\end{array}$ & $P$ value \\
\hline Disc diffusion & 0.61 & Good agreement & 81.08 & $0.000 *$ \\
\hline Flow cytometry & 0.88 & Excellent agreement & 95.59 & $0.000 *$ \\
\hline
\end{tabular}

a: Kappa values: 0.00-0.4, fair agreement ; 0.41-0.75, good agreement ; 0.76-1.00, excellent agreement.

b: Percentage agreement equals the agreement between the standard test (MD) and either disc diffusion or flowcytometry

\section{DISCUSSION}

The increasing number of clinical isolates of oropharngeal Candida albicans resistant to antifungal therapy led to a demand for susceptibility testing of fungi to provide the treating clinician with MIC end points which are reproducible and predictive of therapeutic success or failure. They are only useful clinically if the results are determined after a short incubation period $(13,26)$.

Our study compared three methods for evaluation of the antifungal susceptibility of yeasts to fluconazole: flow cytometry (FCM) assay (after 4h) and disk diffusion (DD) test ( after $24 \mathrm{~h}$ ) in comparison to the standardized anti-fungal susceptibility test method by MD (M27-A2) of oropharyngeal 
Candida albicans strains after $24 \mathrm{~h}$ among some Egyptian haematological malignant patients.

The MD M27-A2 method revealed FZ resistance in 6 (16.2\%) isolates while dose-depending susceptibility occurred in $5(13.5 \%)$.

However, Enwuru et al., (9) informed that among 30 Candida albicans isolated from oropharyngeal HIV/AIDS patients, 26 (86.7\%) were sensitive to FZ; 1 (3.3\%) was S-DD and $3(10 \%)$ were resistant. Wilheim et al., (27) reported that both, resistance (14.28\%) and dose-depending susceptibility (4.76\%) of esophageal candidiasis to $\mathrm{FZ}$ were considered high among immunocompromized patients.

In the present study, FCM technique, assayed by PI fluorescence emission, provides MD-comparable results for all 37 strains following a short period of incubation (4h).

It had been verified in other studies (21) that antifungal agents to work well with FCM and PI dye needs incubation time that produces clearly definable changes in the FCM density plot and allow for results to be obtained on the same working day. The alterations in the side scatter and forward scatter cytograms, indicative of the antifungal effect on the yeast cells, are also only minimally apparent with a shorter incubation time. The generation time of Candida is approximately $3 \mathrm{~h}(20)$, so it is understandable that the drug effects take at least a partial growth cycle to be detected.

We found mean channel fluorescence (MCF) to be a reliable parameter for all 37 strains tested. This is because FCM measures antibiotic-induced membrane damage. We used sodium deoxycholate to enhance diffusion of PI across the cell wall to pass through the damaged yeast cell membranes as recommended previously (21).

Most previous studies of FZ defined the parameters of MIC by FCM as the drug concentration that produced a $50 \%$ increase in MCF $(11,19)$. However, Joung et al. (12) demonstrated that a $50 \%$ increase in MCF might not be the best criterion for determining the MIC of FZ and recommended, a $30 \%$ increase in MCF was best correlated to the MD MIC under their test conditions that utilized sodium deoxycholate to enhance PI penetration in $8 \mathrm{~h}$ incubation.

For the 37 isolates tested, $89 \%$ of the FCM results were within the same dilution of the MD results, and $100 \%$ of the results were within 2 dilutions. Moreover, the FCM method produced an excellent level of agreement with M27-A. The overall agreement for the MICs between the two methods was 95.59\% for FZ. The agreement between MD and FCM methods ranged from 96 to $99 \%$ by Chaturvedi et al.,(2).

The antifungal disk diffusion method (DD) shows simplicity and flexibility of testing making it a very appealing method for use in the clinical laboratory (18).

By using DD method, the majority of Candida strains (24/37) are susceptible to FZ and had a zone diameter above the breakpoint $(\geq 19 \mathrm{~mm})$, and these strains could safely be reported as susceptible. However, (2 / 37) susceptible strains, for which the MIC was $8 \mu \mathrm{g} / \mathrm{ml}$, were be reported as one resistant and one as S-DD by the DD. Therefore, the detection of resistance by disk test correlates poorly with that by the reference MD M27-A2 method. We could say that interpretation of resistant cases by DD method should be taken cautiously. This is similar to a study by Matar et al., (14) who concluded that $\geq 90 \%$ of the isolates that tested resistant to FZ by an agar-based method were susceptible-dose dependent or resistant by the reference MD method

In our study, the agreement between the DD and MD methods was $81 \%$ better than that reported previously (17) as being $67 \%$. The DD zone diameters are highly reproducible and correlate well with the MD method, making agar-based method a viable alternative to MD for susceptibility testing.

Considering that DD assays are simple to perform and inexpensive, they may be a useful tool in large-scale surveys of clinical isolates to identify population distribution patterns of FZ sensitivity of Candida albicans strains (8). FZ MICs should be determined for strains with zone diameters below the breakpoint ( $\leq 19 \mathrm{~mm}$ ). The reason for confirmatory testing, as explained by Sandven (25), is twofold: to determine if isolates 
are resistant or S-DD, since the FZ disc test does not make this distinction, and to identify FZ-susceptible strains that are found to be falsely resistant by the disc test.

In conclusion, The FCM method using PI generally provided rapid (4h) and reproducible results instead of the $24 \mathrm{~h}$ required by broth MD. However, when the number of simultaneously tested samples is increased, this procedure becomes quite labor-intensive, which limits its use in routine laboratories. DD assay for FZ susceptibility testing is simple to perform and inexpensive. It may be a useful tool in large-scale surveys with taken cautiously the interpretation of resistant isolates by DD.

\section{REFERENCES}

1. Barry, A.L.; Brown, S.D. (1996) Fluconazole disk diffusion procedure for determining susceptibility of Candida species. J Clin Microbiol. 34(9):2154-2157

2. Chaturvedi, V.; Ramani, R.; Pfaller, M.A. (2004). Collaborative study of the NCCLS and flow cytometry methods for antifungal susceptibility testing of Candida albicans. J. Clin. Microbiol. 42(5), 2249-2251.

3. Clinical and Laboratory Standards Institute (2006). Quality control minimal inhibitory concentration (MIC) limits for broth microdilution and MIC interpretive breakpoints. Supplement M27-S2. Clinical and Laboratory Standards Institute, Wayne, PA.

4. Clinical and Laboratory Standards Institute (2008). Reference method for broth dilution antifungal susceptibility testing of yeasts. Approved standard, 3rd ed. M27-A3. Clinical and Laboratory Standards Institute, Wayne, PA.

5. Clinical and Laboratory Standards Institute (2006). Zone diameter interpretive standards and corresponding minimal inhibitory concentration (MIC) interpretive breakpoints, Supplement M44-S1. Clinical and Laboratory Standards Institute, Wayne, PA

6. Clinical and Laboratory Standards Institute/National Committee for Clinical Laboratory Standards (2002). Reference method for broth dilution susceptibility testing of yeasts. Approved Standard, 2nd ed. Document M27-A2. National Committee for Clinical Laboratory Standards, Wayne, PA.

7. Clinical and Laboratory Standards Institute/National Committee for Clinical Laboratory Standards (2004). Method for antifungal disk diffusion susceptibly testing of yeasts. Approved guideline. Document M44-A. National Committee for Clinical Laboratory Standards, Wayne, PA
8. Colombo, A.L.; Da Matta, D.; De Almeida, L.P.; Rosas, R. (2002). Fluconazole susceptibility of Brazilian Candida isolates assessed by a disk diffusion method. Braz J. Infect. Dis. 6 (3), 118-123.

9. Enwuru, C.A.; Ogunledun, A.; Idika, N.; Enwuru, N.V.; Ogbonna, F.; Aniedobe, M.; Adeiga, A. (2008). Fluconazole resistant opportunistic oro-pharyngeal Candida and non-Candida yeast-like isolates from HIV infected patients attending ARV clinics in Lagos, Nigeria. Afr. Health. Sci. 8(3), 142-148.

10. Espinel-Ingroff, A.; Canton, E.; Gibbs, D.; Wang, A. (2007). Correlation of Neo-Sensitabs tablet diffusion assay results on three different agar media with CLSI broth microdilution M27-A2 and disk diffusion M44-A results for testing susceptibilities of Candida spp. and Cryptococcus neoformans to amphotericin B, caspofungin, fluconazole, itraconazole, and voriconazole. J. Clin. Microbiol. 45(3), 858-864.

11. Gokahmetoglu, S.; Nedret Koc, A.; Patiroglu, T. (2003). Antifungal susceptibility testing of Candida albicans by flowcytometry. Mycoses. 46, 289-293.

12. Joung, Y.H.; Kim, H.R.; Lee, M.K.; Park, A.J. (2007). Fluconazole susceptibility testing of Candida species by flowcytometry. J. Infect. 54(5), 504-508.

13. Koga-Ito, C.Y.; Lyon, J.P.; Resende, M.A. (2008). Comparison between E-test and CLSI broth microdilution method for antifungal susceptibility testing of Candida albicans oral isolates. Rev. Inst. Med. trop. S. Paulo. 50(1), 7-10.

14. Matar, M.J.; Ostrosky-Zeichner, L.; Paetznick, V.L.; Rodriguez, J.R.; Chen, E. Rex, J.H. (2003). Correlation between E-test, disk diffusion, and microdilution methods for antifungal susceptibility testing of fluconazole and voriconazole. Antimicrob. Agents. Chemother. 47(5), 1647-1651.

15. Million, L.; Manteaux. A.; Reboux, G.:, Drobacheff, C.; Monod, M.; Barale, T.; Michel-Briand, Y. (1994). Fluconazole- resistant recurrent oral candidiasis in human immuno-deficiency Virus positive patients: persistence of Candida albicans strains with the same genotype. J. Clin. Microbiol. 32 (4), 1115 - 1118.

16. Mitchell, M.; Hudspeth, M.; Wright, A. (2005). Flowcytometry susceptibility testing for the antifungal caspofungin. J. Clin. Microbiol. 43(6), 2586-2589.

17. Negri, M.; Henriques, M.; Svidzinski, T.I.; Paula, C.R.; Oliveira, R. (2009). Correlation between $\mathrm{E}$ test, disk diffusion, and microdilution methods for antifungal susceptibility testing of Candida species from infection and colonization. J. Clin. Lab. Anal. 23(5), 324-330.

18. Pfaller MA, Boyken LB, Hollis RJ, Kroeger J, Messer SA, Tendolkar S, Diekema DJ. (2008). Validation of 24-hour fluconazole MIC readings versus the CLSI 48-hour broth microdilution reference method: results from a global Candida antifungal surveillance program. $J$ Clin Microbiol.46(11), 3585-3590. 
19. Pina-Vaz, C.; Sansonetty, F.; Rodrigues, A.G.; Costa-Oliveira, S.; Tavares, C.; Martinez,-de-; Oliveira, J. (2001). Cytometric approach for a rapid evaluation of susceptibility of Candida strains to antifungals. Clin Microbiol. Infect.7(11), 609-618.

20. Pore, R.S. (1994). Antibiotic susceptibility testing by flowcytometry. $J$. Antimicrob.Chemother. 34, 613-627.

21. Ramani, R.; Ramani, A.; Wong, S.J. (1997). Rapid flowcytometric susceptibility testing of Candida albicans. J. Clin. Microbiol. 35(9), 2320-2324.

22. Ramani, R.; Chaturvedi, V. (2000). Flowcytometry antifungal susceptibility testing of pathogenic yeasts other than Candida albicans and comparison with the NCCLS broth microdilution test. Antimicrob. Agents. Chemother. 44, 2752-2758.

23. Rimek, D.; Redetzke, K.; Kappe, R. (2006). Impact of antifungal prophylaxis on the gastrointestinal yeast colonization in patients with hematological malignancies Mycoses. 49 Suppl 2, 18-23
24. Rudensky, B.; Broidie, E.; Yinnon, A.M.; Weitzman, T.; Paz, E.; Keller, N.; Raveh, D. (2005). Rapid flowcytometric susceptibility testing of Candida species. J. Antimicrob. Chemother. 55(1), 106-109.

25. Sandven, P. (1999). Detection of fluconazole-resistant Candida strains by a disc diffusion screening test. J. Clin. Microbiol. 37(12), 3856-3859.

26. Wenisch, C.; Moore, C.B.; Krause, R.; Presterl, E.; Pichna, P.; Denning, D.W. (2001). Antifungal susceptibility testing of fluconazole by flowcytometry correlates with clinical outcome. J. Clin. Microbiol. 39(7), 2458-2462.

27. Wilheim, A.B.; Miranda-Filho Dde, B.; Nogueira, R.A.; Rêgo, R.S.; Lima, K.de M.; Pereira, L.M. (2009). The resistance to fluconazole in patients with esophageal candidiasis. Arq. Gastroenterol. 46(1), 32-37.

28. Yu, D.T.; Seger, D.L.; Peterson, J.F.; Kumar, R.N.; Bates, D.W. (2006). Fluconazole for empiric antifungal therapy in cancer patients with fever and neutropenia. BMC. Infect. Dis. 5(6), 173-183. 